\title{
Complete nucleotide sequence of the A+T-rich region of Drosophila mauritiana mitochondrial DNA
}

\author{
Kanako Sugihara $^{1 \dagger}$, Ryoko Yui ${ }^{2}$, Yoko Ibaragi ${ }^{1}$ and Etsuko T. Matsuura ${ }^{3 *}$ \\ ${ }^{1}$ Department of Molecular Biology \& Biochemistry, \\ ${ }^{2}$ Department of Advanced Biosciences, \\ ${ }^{3}$ Department of Biology, \\ Ochanomizu University, Otsuka, Bunkyo-ku, Tokyo 112-8610, Japan
}

(Received 21 September 2005, accepted 19 December 2005)

\begin{abstract}
We determined the complete nucleotide sequence of the A+T-rich region of the maII type of mtDNA in D. mauritiana. The nucleotide sequence was found to contain 3,206 bp. Three types of conserved element, i.e., type I element, type II element, and T-stretch, were included in this sequence, as reported for $D$. melanogaster. Comparison between the two species revealed that the type I elements were less conserved than the type II elements. However, each of these type I elements contained a G-stretch within a loop of a putative stem-loop-forming sequence, which has also been observed in D. melanogaster. Moreover, in both type I and type II repeat arrays, the elements closest to the T-stretch diverged the most, due to nucleotide substitution and/or the insertion of short repeats. Sequence comparison of the two complete sequences of the A+T-rich region of $D$. melanogaster and the maII type of $D$. mauritiana, as well as comparison of partial sequences in other types of mtDNA within the melanogaster complex, suggested that the A+T-rich region in this complex has been maintained by concerted evolution after the duplication of two types of element, i.e., type I and type II.
\end{abstract}

Key words: Drosophila mauritiana, A+T-rich region, repeats

\section{INTRODUCTION}

The control region in mitochondrial DNA (mtDNA) is consisted of a single large non-coding region of mitochondrial genome in metazoan animals. In spite of its high variability with respect to nucleotide sequences, the control region is necessary for the initiation of replication and transcription of mtDNA (Shadel and Clayton, 1997). To date, most studies of this region have focused on mammalian mtDNA. In insects, the control region contains nearly $90 \%$ adenine (A) and thymine (T), and thus is referred to as an A+T-rich region. This region has been analyzed in crickets (Rand and Harrison, 1989), honeybees (Crozier and Crozier, 1993), grasshoppers (Zhang et al., 1995), moths (Snall et al., 2002), mosquitoes (Caccone et al., 1996), and flies (Clary and Wolstenholme, 1985; Monnerot et al., 1990; Monforte et al., 1993; Lewis et al., 1994; Brehm et al., 2001), as well as in other insects. These previous studies have identified the sequence of the Ts (T-stretch) and the putative secondary

Edited by Yoko Satta

* Corresponding author: E-mail: etmatsu@cc.ocha.ac.jp

$\dagger$ Present address: Data Management Department, Biometrics Division, EPS Co., Ltd. structures in the A+T-rich region. In Drosophila, the replication of mtDNA has been suggested to start at the center of the A+T-rich region, as determined by electron microscopy (Goddard and Wolstenholme, 1978, 1980). Saitou et al. (2005) successfully determined the replication origins of mtDNA in several insect species, and discussed the evolution of functional elements within this region.

The size of the A+T-rich region is typically approximately $1 \mathrm{~kb}$ (short) in most Drosophila species thus far examined. In the case of $D$. melanogaster and its closely related species, $D$. mauritiana, $D$. simulans, and $D$. sechellia, in which eight types of mtDNA have been reported (Solignac et al., 1986a), the size of the A+T-rich regions in these species is exceptional, namely, their A+T-rich regions range from about 4 to $5 \mathrm{~kb}$ in length. Among these species, $D$. melanogaster is the only one for which the entire mtDNA nucleotide sequence has been determined, and three types of elements, i.e., type I element, type II element, and T-stretch, have been identified (Lewis et al., 1994). One-half of the A+T-rich region adjacent to the tRNA ${ }^{\text {ile }}$ gene is known to be comprised of five type II elements, and the other half of the A+T-rich region is also comprised of five type I elements. Two T- 
stretches are present in this region; one of these Tstretches is adjacent to the gene for tRNA ${ }^{\text {ile }}$, and the other is at the center of the A+T-rich region in the opposite strand.

To date, the nucleotide sequences of only two portions of the A+T-rich region in species closely related to $D$. melanogaster have been examined; it has been demonstrated that these portions also possess these three types of element (Inohira et al., 1997; Tsujino et al., 2002). The nucleotide sequences of the type II elements are relatively well conserved among Drosophila species. A sequence of approximately $280 \mathrm{bp}$ in length within the type II element is highly conserved, and this element is therefore referred to as a HCSE (Highly Conserved Sequence Element). Restriction analyses have confirmed the repeated structure of this conserved region (Solignac et al., 1986b). In contrast, the sequences of the other half of the A+T-rich region vary among species (Lewis et al., 1994). The formation of heteroduplexes between mtDNA molecules derived from different species was observed in the conserved region adjacent to the tRNA ${ }^{\text {ile }}$ gene, but such heteroduplexes have not been observed in this variable region (Fauron and Wolstenholme, 1976). Although we previously identified the type I elements in the melanogaster complex, the complete array of the repetition of type I elements has not yet been reported.

To gain a better understanding of the evolution of the repeated structures and the functional sequences within the A+T-rich region, it will be necessary to analyze its entire sequence in species other than $D$. melanogaster that exhibit a long A+T-rich region. In the present study, we determined the complete nucleotide sequence of the A+T-rich region of D. mauritiana (maII type) and compared this sequence with the corresponding sequence of D. melanogaster. This is the first report to provide a comparative analysis of the repeated sequences together with that of the entire A+T-rich region in Drosophila.

\section{MATERIALS AND METHODS}

Fly strain An isofemale strain of $D$. mauritiana, the g52 strain, which was established from a single female from a wild population in Mauritius, was used in the present study. The mitochondrial genome of the g52 strain was examined by restriction analyses and determined to be of the maII type according to the Solignac et al. (1986a).

Cloning and Sequencing Mitochondrial DNA was extracted from adult flies as previously described (Satta et al., 1987). A 4.4-kb HindIII fragment containing the entire A+T-rich region (Solignac et al. 1986a) was cut by $H p a \mathrm{I}$ into two fragments, a 0.8-kb HindIII/HpaI fragment and a 3.6-kb HindIII/HpaI fragment. These fragments were purified by agarose gel electrophoresis and were collected using a QIAEX II Gel Extraction Kit (QIAGEN). The 0.8-kb HindIII/HpaI fragment was directly ligated into pTV118N (TaKaRa), which was digested by HindIII and HincII. The 3.6-kb Hin $\mathrm{dIII} / \mathrm{HpaI}$ fragment was partially digested by AseI, and each fragment was then cloned into pUC118N HincII/BAP (TaKaRa).

We also conducted PCR in order to confirm that only one $\mathrm{HpaI}$ site was present, as previously reported (Solignac et al., 1986b); for the PCR, the following oligonucleotides were used as primers: 5'-GGTGTATGATGCACAAAG-3' and 5'-CTGATAACTTATTCCCCTA-3', which were designed based on the nucleotide sequences of $D$. yakuba mtDNA (Clary and Wolstenholme, 1985). The reaction was performed in $50 \mu \mathrm{l}$ of solution containing 50 $\mathrm{mM} \mathrm{KCl}, 10 \mathrm{mM}$ Tris-HCl (pH 8.3), 0.1\% Triton X-100, $2.0 \mathrm{mM} \mathrm{MgCl}, 160 \mu \mathrm{M}$ of each dNTP, $10 \mathrm{pmol}$ of each primer, approximately $200 \mathrm{ng}$ of the template DNA, and $2.5 \mathrm{U}$ of rTaq DNA polymerase (TOYOBO). The reaction conditions were as follows: $5 \mathrm{~min}$ at $94^{\circ} \mathrm{C}$ followed by 30 $\mathrm{s}$ at $94^{\circ} \mathrm{C}, 30 \mathrm{~s}$ at $30^{\circ} \mathrm{C}$, and $90 \mathrm{~s}$ at $60^{\circ} \mathrm{C}$ for 30 cycles. The PCR products were purified using QIAquick PCR Purification Kit (QIAGEN), and then the products were cloned into a pGEM ${ }^{\circledR}$-T easy vector using the pGEM-T easy vector system I (Promega).

The determination of the nucleotide sequences was carried out at least three times for each clone by the cycle sequencing method, using Thermo Sequence Fluorescent Labeled Primer Cycle Sequencing Kit (Amersham Pharmacia Biotech) and BigDye ${ }^{\circledR}$ Terminator v3.1 Cycle Sequencing Kit (Applied Biosystems) by the automated sequencer DSQ-2000L (SHIMADZU) and ABI Prizm ${ }^{\circledR}$ 3100-Avant (Applied Biosystems), respectively.

Sequence Analysis The sequences were mapped on the A+T-rich region according to previously analyzed sequences from other mtDNA types (Tsujino et al., 2002). The sequences were aligned with the corresponding sequences of $D$. melanogaster (Lewis et al., 1994) by GENETIX software (Version 9.0) and CLUSTAL W (Thompson et al., 1994), as well as by eye. The number of nucleotide substitutions per site was estimated by the $p$-distance (Nei and Kumar, 2000). Neighbor-joining (NJ) trees (Saitou and Nei, 1987) based on the $p$-distances were constructed for the type I and type II elements within the A+T-rich region using MEGA (version 2.1) (Kumar et al., 2001). To predict the stem-loop-forming sequences, mfold (version 3.1) was used (Zuker, 2003, http:/ / www.bioinfo.rpi.edu / applications / mfold/old/ dna).

The sequence determined in the present study is deposited in the DDBJ with the accession number AB242844. 


\section{RESULTS}

The A+T-rich region of the maII type consisted of 3,206 $\mathrm{bp}$, and the content of $\mathrm{A}+\mathrm{T}$ was $95.0 \%$.

The organization of the entire A+T-rich region of maII is illustrated in Fig. 1. Three types of conserved DNA elements were found. The first one was a T-stretch; one such element was located adjacent to the tRNA ${ }^{\text {ile }}$ gene, and the other was at the center of the A+T-rich region in the opposite strand. The second type of element was a type II element containing an HCSE. Two type II elements were identified in maII, one of which was $465 \mathrm{bp}$ in length and was adjacent to the tRNA ${ }^{\text {ile }}$ gene. The other was incomplete, $186 \mathrm{bp}$ in length, and contained a large deletion and numerous substitutions. The A+T content in these two type II elements was $94.4 \%$ and

D. melanogaster ${ }^{\text {TRNA }}$ ile 1

\section{D. mauritiana (mall)}

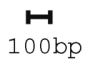

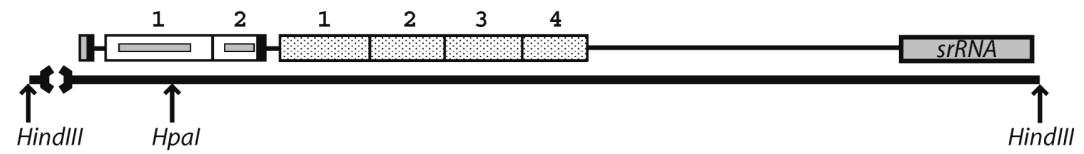

Fig. 1. The organization of the A+T-rich region of D. mauritiana (maII). The corresponding region of D. melanogaster is also indicated (Lewis et al. 1994). The open boxes with gray bars indicate the type II elements containing the highly conserved sequence elements. The dotted boxes indicate the type I elements.

mel 1 AaATAATACATTTAAGAAATTTTTTAAAATTTTATGAAAAATTATTTAAATAATTTTATTTTTCTATATATATATATATT-ATATATAAATATTCAATAA mel 2 AAATAATACATTTAAGAAATTTTAAAAAATTTATATTAAATTATTTAAATAATTTAATTTTCTATATATATATATATA-TTATATAAATATTCAATAA mel 3 AAATAATACATTTAAGAAATTTTAAAAAATTTATATTAAATTATTTAAATAATTTAATTTTTCTATATATATATATATA-TTATATAAATATTCAATAA mel 4 AAATAATACATTTAAGAAATTTTAAAAAATTTATATTAAATTATTTAAATAATTTAATTTTTCTATATATATATATATA-TTATATAAATATTCAATAA mel 5 AAATAATACATTTAAGAAATTTTTAAAAAATTTATATTAAATTATTTAAATAATTTAATTTTTCTATATATATATATATA-TTATA--AATATTCAATAA maI I 1 AAATAATACTTTTAAGAATAAATTAAAAATATTATAAAAAATTATTAAGATAATTTAATTTTTCTATATATATATATATG-TTATATAAATATTCAATAA

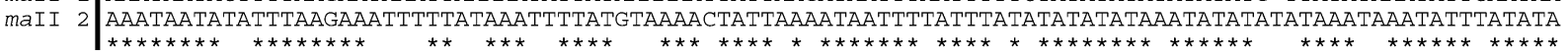
200 mel 1 TATATAAATTTATAAATATATAATAATTAATTAAATCATTATATTATTTATATAAATTAAATTAATAATAAATAAATATGAGAATATAAA- - - - - TTTT mel 2 TATATAAATTTATAAATATATAATAATTAATTAAATCATTATATTATTTATATAAATTAAATTAATAATAAATAAATATGAGAATATAAA- - - - - TTTT mel 3 TATATAAATTTATAAATATATAATAATTAATTAAATCATTATATTATTTATATAAATTAAATTAATAATAAATAAATATGAGAATATAAA- - - - - TTTT mel 4 TATATAAATTTATAAATATATAATAATTAATTAAATTATTATATTATTTATATAAATTAAATTAATAATAAATAAATATGAGAATATAAA- - - - - TTTT mel 5 TATATAAATTTATAAATATATAATAATTAATTAAATTATT $-\ldots \ldots \ldots \ldots$ maII 1 TATATAAATTTATAAATATATAATAATTAATTAAATTATTATATTATTTATATAAATCCAA-GAATAATAGATAAATATATTAATATAAA- - - - -TTTT

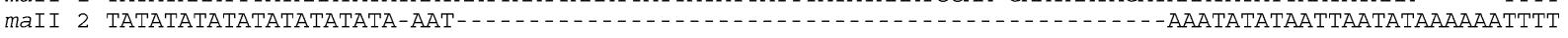

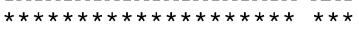

300

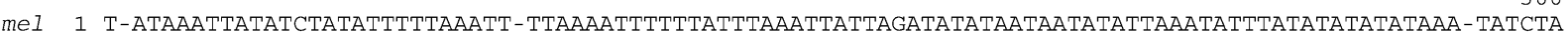
mel 2 T-ATAAATTATATCTACATTTTAAATT-TTAAAATTTTTATTTAAATTATTAGATATATAATAATATATTAAATATTTATATATATATAAA-TATCTA

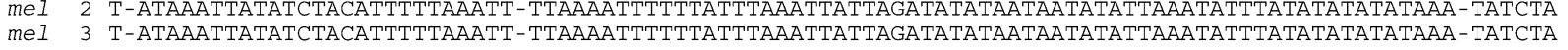
mel 4 T-ATAAATTATATCTACATTTTTAAATT-TTAAAATTTTTTATTTAAATTATTAGATATATAATAATATATTAAATATTTATATATATATAAA-TATCTA

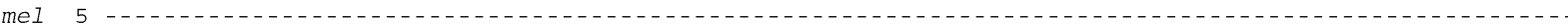
maII 1 T-ACAAATTATGTTTACATTTTTGGATTGTGAAA-TTTTTTATTTAAATTGTTAACTATATTATAATATATTAAATATTTATATATATATAGATTATCTA

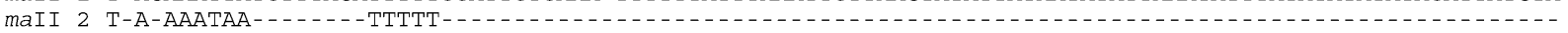

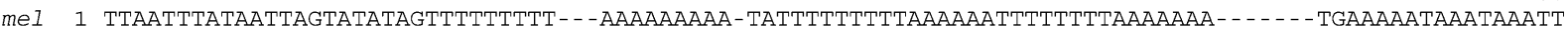
mel 2 TTAATTTATAATTAGTATATAGTTTTTTTTT--AAAAAAAAAATTATTTTTTTT-AAAAAATTTTTT- -AAAAAAA- - - - - TTGAAAAATAAATAAATT mel 3 TTAATTTATAATTAGTATATAGTTTTTTTTT--AAAAAAAAAATTATTTTTTTT-AAAAAATTTTTT--AAAAAAA- - - - TTGAAAAATAAATAAATT mel 4 TTAATTTATAATTAGTATATAGTTTTTTTTT--AAAAAAAAAATTATTTTTTTT-AAAAAATTTTTTTTT-AAAAA-- - - - - TGAAAAATAAATAAATT mel 5 maII 1 TTAATTTATAACTAGTATACAATTTTTTATTTTTAAAAAAAAA-TATTTTTT---AAAAAATTTTTT--AAAAAAATATTTTTTAAAAAATAAATAAAA-

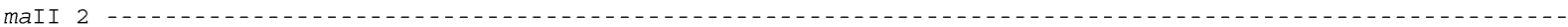

487

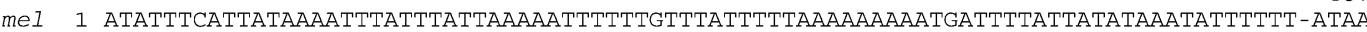
mel 2 ATATTTCATTATAAAATTTATTTATTAAAAATTTTTTGTTTATTTTTTAAAAAACATGATTTTATTATATAAATATTTTTT-ATAA

mel 3 ATATTTCATTATAAAATTTATTTATTAAAAATTTTTTGTTTATTTTTTAAAAAACATGATTTTATTATATAAATATTTTTT-ATAA mel 4 ATATTTCATTATAAAATTTATTTATTAAAAATTTTTTGTTTATTTTTTAAAAAACATGATTTTATTATATAAATATTTTTT-ATAA mel 5

maII 1 ---TTTCATTATAATTATTTTTTATTAAAAATTTTGTTTATTTTTAATAAAAAATTATTTTTTTATATAAATATTTTTTTATAT

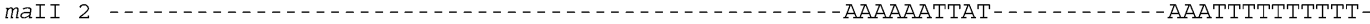

Fig. 2. Alignment of the nucleotide sequences of the type II elements of D. melanogaster and of D. mauritiana. A dash represents a nucleotide that is absent. Asterisks indicate nucleotide sites at which the nucleotides were identical among the elements compared. 
$98.4 \%$, respectively. The alignment of the nucleotide sequences of the type II elements of $m a \mathrm{II}$ and those of $D$. melanogaster (Lewis et al., 1994) is shown in Fig. 2. Five type II elements were found in D. melanogaster, and the outermost element adjacent to the tRNA ${ }^{\text {ile }}$ gene contained many nucleotide substitutions, and the innermost element was also incomplete. Excluding these unusual elements, the alignment indicated that the type II elements are well conserved between maII and $D$. melanogaster.

The third type of element identified here was a type I element, which was repeated tandemly, as previously reported in D. melanogaster (Lewis et al., 1994). The alignment of the four type I elements of $m a \mathrm{II}$ and the five type I elements of D. melanogaster is shown in Fig. 3. As regards the size of the type I elements, it ranged from a length of $263 \mathrm{bp}$ to $386 \mathrm{bp}$, and the A+T-content ranged from $92.8 \%$ to $95.6 \%$. Moreover, the type I elements were less conserved than the type II elements. The average $p$-distance between the type I elements of maII and D. melanogaster, 0.250 , was twice that of the type II elements, 0.134. The first element of the type I array, i.e., the innermost element, included two short repeat elements near the T-stretch and its repeat unit was 45 -bp long. The fourth element, i.e., the outermost element, contained a 66-bp deletion. As regards the A+T-rich region of $D$. melanogaster containing five type I elements, more nucleotide substitutions were observed in the first element than in the other elements, and short repeat elements were present in the region between the central Tstretch and the first element. Moreover, the type I elements of both maII and D. melanogaster contained a stretch of four and five Gs, respectively.

It was of note that the number of nucleotides between the type I element and the srRNA gene was relatively large in the maII type of mtDNA, namely, there were $1,136 \mathrm{bp}$ in this region in maII, and $649 \mathrm{bp}$ in $D$. melanogaster. The alignment of this region of these two mtDNA types is shown in Fig. 4. A G-stretch was found only in the maII type of mtDNA, and the flanking sequences of this G-stretch were not homologous to those in the type I element.

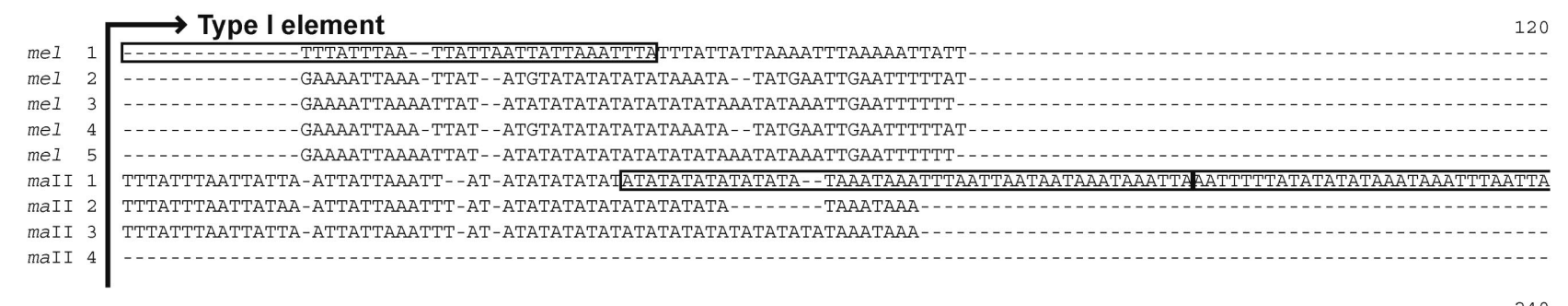

120

240

$\begin{array}{lll}\text { mel } & 1 & - \text {-TTCATTTTAATATATATATATAT-ATA-TATATATAATTTTTATTAATTATTTTAAATAATTT-TATTTATAAAATAATTTATTATAAAAATAGTTTATTAAGTATA-ATTTAATAAA } \\ \text { mel } & 2 & \text {--AAAAAATCATTTTAAATTTTATTATATTAAAAATATTT-TTATTAATTATTTAAAATAATTT-TATTTATAAAATAATTATTATAAAAATAGTTATTAAGTATA-ATTTAATAAA }\end{array}$

mel 3 -- -AAAAATTATTTTTAATTTTTATTATAATAAAAATATTTCTTATTAATTATTTTAAATAATTT-TATTTATAAAATAATTTATTATAAAAATAGTTTATTAAGTATA-ATTTAATAAA

mel 4 --AAAAAATCATTTTAAATTTTTATTATATTAAAAATATTT-TTATTAATTATTTAAAATAATTT-TATTTATAAAATAATTTATTATAAAAATAGTTTATTAAGTATA-ATTTAATAAA

mel 5 ---AAAAATTATTTTTAATTTTTATTATAATAAAAATATTTCTTATTAATTATTTTAAATAATTT-TATTTATAAAATAATTTATTATAAAAATAGTTTATTAAGTATA-ATTTAATAAA maII 1 ATAATAAATAAATTPATTTATTAATATTTATAATATTTATTTTTATTAATTATTTCGGTTAATTTATATATATAAATAAAATTATTATTAAAATAATTTATTAAGATTATACTTAATTTA

maII 2 maII 3 - - - - - TTTATTTATTAATATTTATAATATTTATTTTTATTAATTATTTCGGTTAATTTTTATATATGAATAAAATTATTATTAAAATAATTTATTAAGATTATATTTAATTTA maI 4 -.....- TTATTTATTAATATTTATAATATTTATTTTTATTAATTATTTCGGTTAATTTATATATATAAATAAAATTATTATTAAAATAATTTATTAAGATTATATTTAATTTA $* \quad * \quad * * * * * * * * * * * * * * * * * * * * \quad * * * * * * * * * * * * * * * * * * * * * * * * * * * * * * * * * * * * * * * * * *$

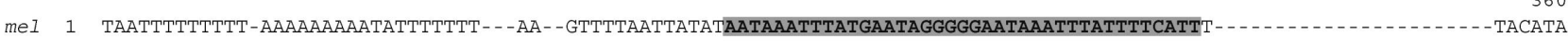

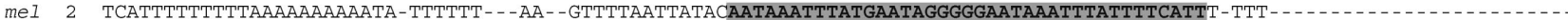

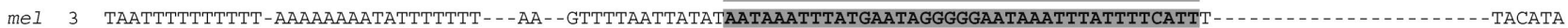

mel 4 TCATTTTTTTTTAAAAAAAAAATA-TTTTTT--- AA-- GTTTTAATTATACAATAAATTTATGAATAGGGGAATAAATTTATTTTCATTT-TTT- - - - - - - - - - - - - - - - - - - - -

mel 5 TAATTTTTTTTT-AAAAAAAAATAC-TTTTT---AA--GTTTTAATTATATAATAAATTTATGAATAGGGGGAATAAATTTATTTTTATTTATTTATGTATATATATATATATATATGTA

maI I 1 TAGTTAAAT-- - AAAATTTATTTATTATTAAAAAAA-GTTTTAATTAT--AATAAAATTATAAATAGGGG--ATAAATTT-

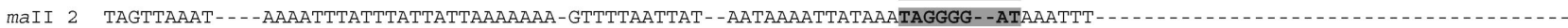

maII 3 TATTTAAAT-.--AAAATTTATTAATTATTAAAAAAA-GTTTTAATTAT--AATAAAATTATAAATAGGGG--ATAAATTT-...............................

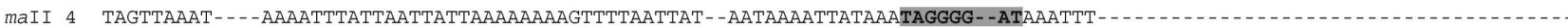

* ** $\quad * * * * * * * * \quad * * * * * * * * * * * * * * * * * * * * * * * * * * * * * * * * * * * *$

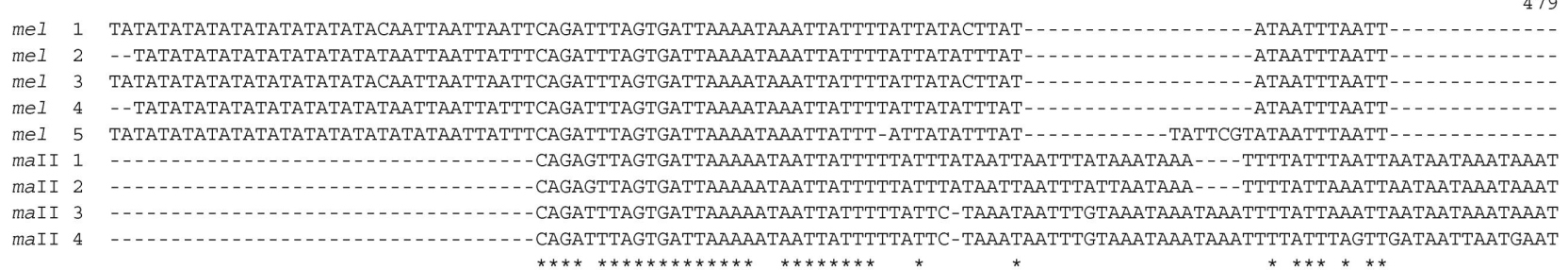

Fig. 3. Alignment of the nucleotide sequences of the type I elements of D. melanogaster and of D. mauritiana. The sequences are shown as described in Fig. 2. Sequences capable of forming stem-loop structures are indicated in bold letters within shaded boxes. The boxed sequences indicate repeat units. 


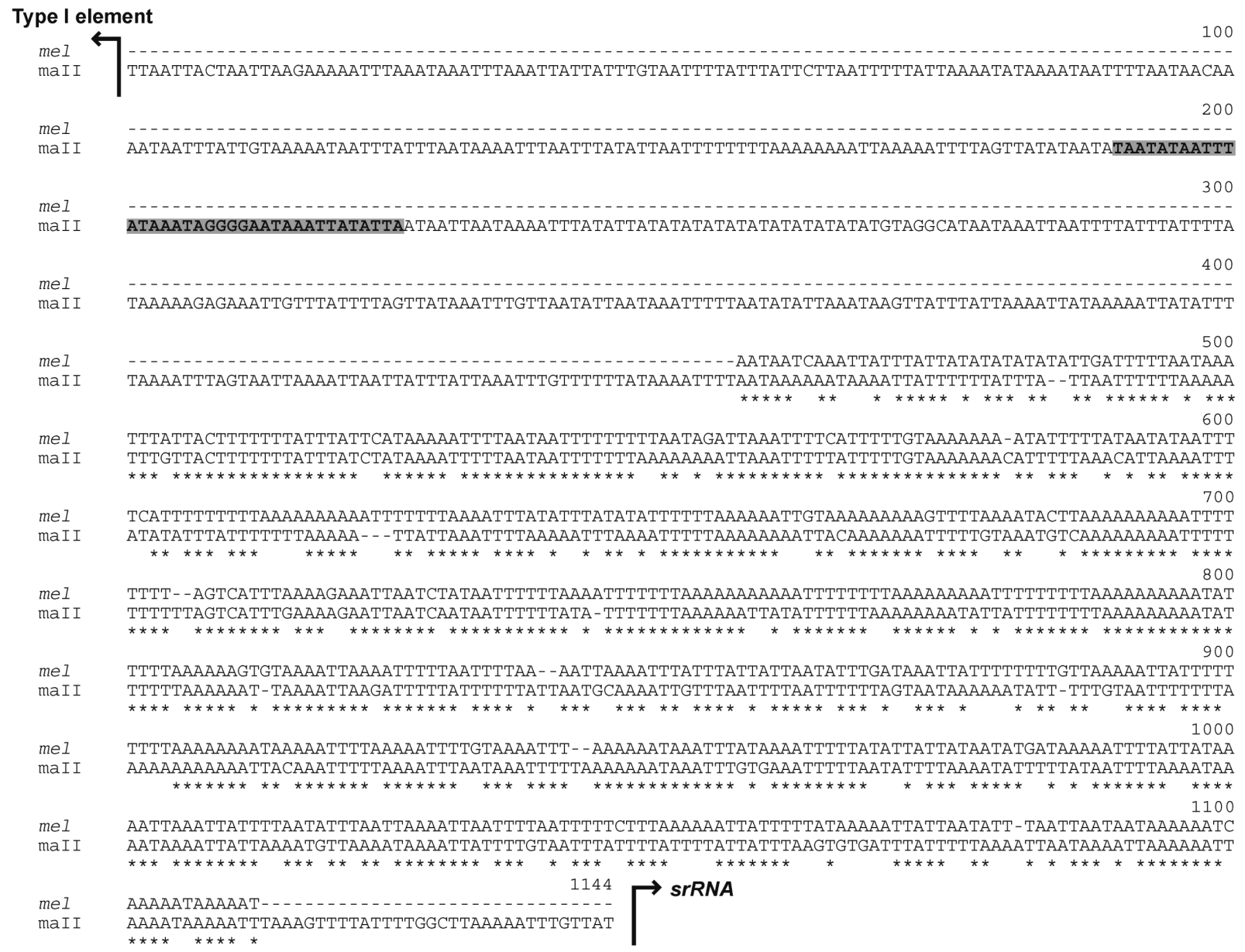

Fig. 4. Alignment of the nucleotide sequences of regions downstream of the type I elements. The sequences are shown as described in Fig. 3.

\section{DISCUSSION}

Conserved motif in the type I element and its downstream region The sequence analysis of the entire A+T-rich region of $D$. mauritiana (maII) revealed for the first time that the type I elements exist as tandem repeats in a species other than $D$. melanogaster (Lewis et al., 1994). Comparison of the species showed that the type I elements were not as conserved as the type II elements. This has also been suggested for other closely related species, as based on partial sequences (Inohira et al., 1997; Tsujino et al., 2002). The results of the present study revealed certain characteristics unique to the type I element. In spite of its high A+T-content, the repetition of Gs was observed in every type I element in the maII type of mtDNA, as well as in that of D. melanogaster. The maII type had an additional G-stretch downstream of the type I element. It should be noted that the mfold program predicted the presence of stem-loop-structures in the vicinity of these G-stretches, and each putative secondary structure had Gs within this loop (Fig. 5). A similar structure has been predicted in the case of (a)

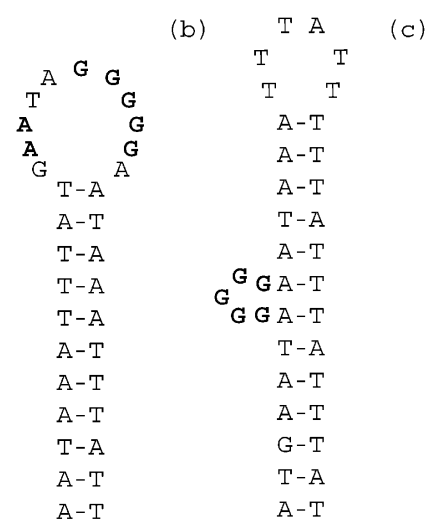

100 00

\section{0}

00 00 AA ** * * TT 00 AA 00 
mary origin of replication (Brehm et al., 2001). It is thought that the replication of mtDNA molecules starts immediately downstream of the T-stretch in the center of the A+T-rich region in Drosophila, as well as in that of certain insect species (Saitou et al., 2005); however, the termination of primary strand replication has not yet been fully elucidated. In mammals and in some other vertebrates, the primary replication of mtDNA is arrested downstream of short conserved sequence elements referred to as TASs (termination-associated sequences) (Saccone et al., 1999). It has been suggested that the termination of replication regulates the mtDNA copy number within cells (Brown and Clayton, 2002). Therefore, the type I elements might be associated with mechanisms important for the maintenance of mtDNA in Drosophila.

Concerted evolution of the A+T-rich region We pre-

(a)

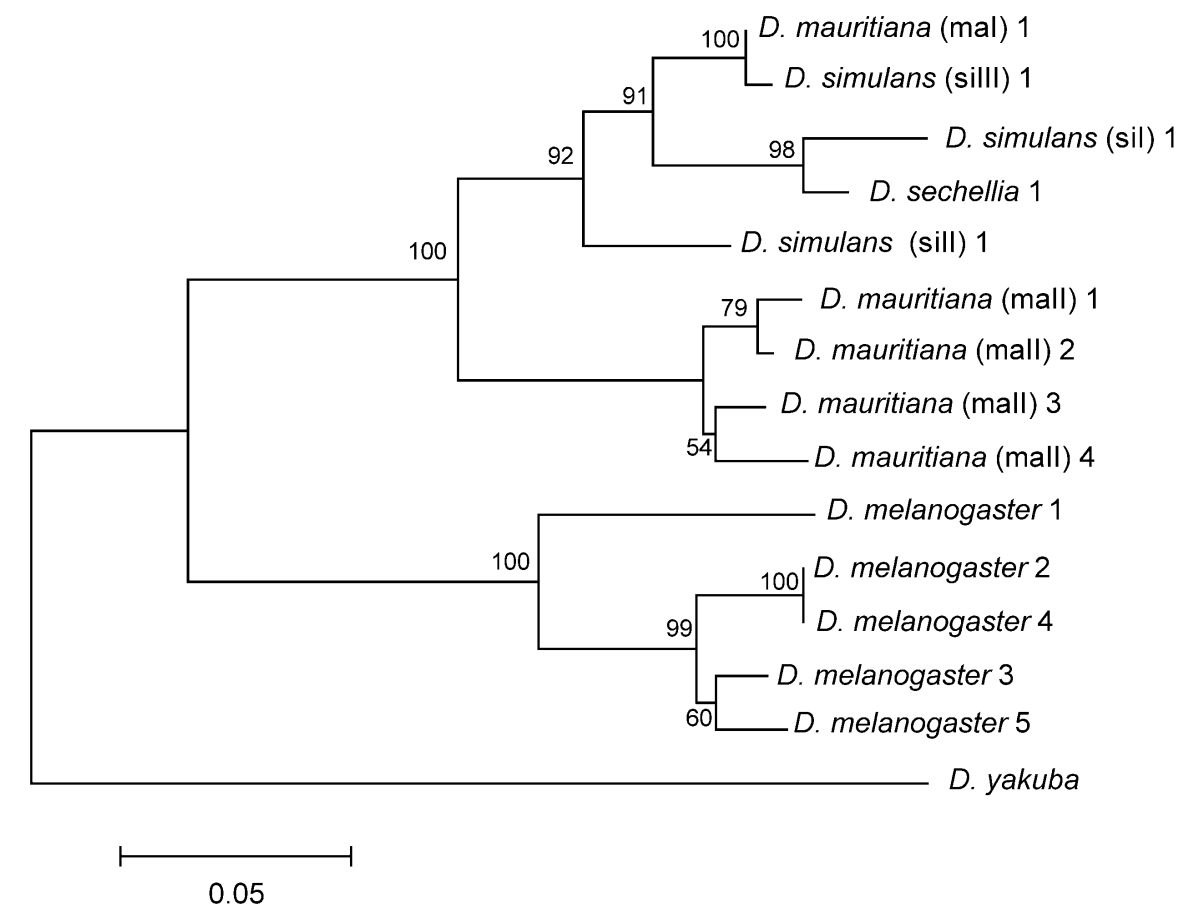

(b)

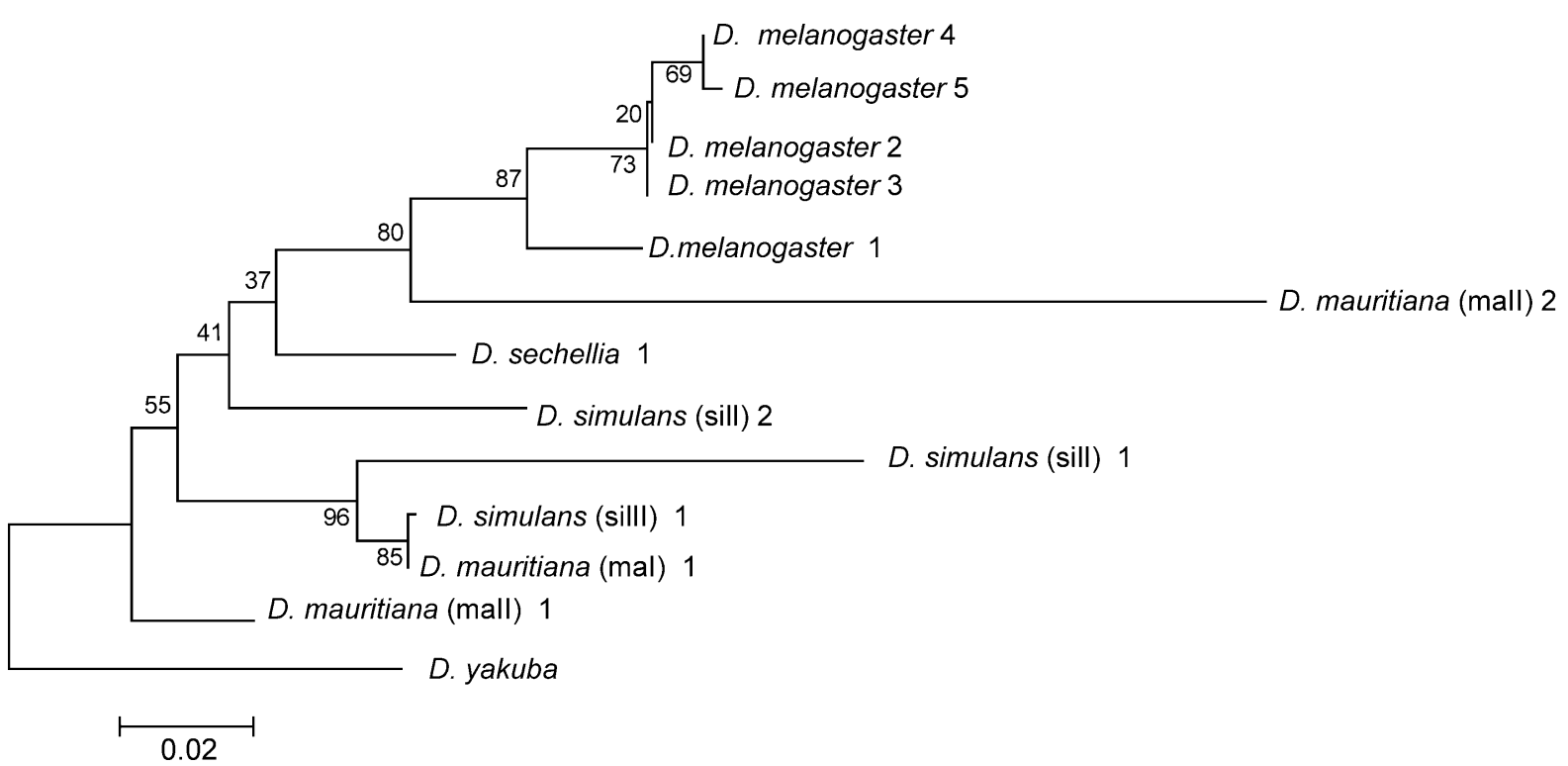

Fig. 6. NJ trees of the sequences of type I elements (a) and type II elements (b). The numbers shown on the branches are bootstrap values based on 500 replicates. 
viously reported the partial sequences of the type I elements and type II elements of other mtDNA types that belong to the melanogaster complex (Inohira et al., 1997; Tsujino et al., 2002). Figure 6a shows the NJ tree based on the alignment of the type I elements among the seven mtDNA types. The topology was consistent with that for the coding sequences of mtDNA (Ballard, 2000), except that the divergence of $D$. simulans (siII) occurred earlier than that of D. mauritiana ( $m a \mathrm{I}), D$. simulans (siI), $D$. simulans (siIII), and D. sechellia. This NJ tree confirms that elements within a single mtDNA type are much more similar to each other than to those of different mtDNA types. A type I element and a type II element were detected in D. yakuba (Inohira et al., 1997), which is a typical species with a short A+T-rich region, and they were also detected in $D$. teissieri (data not shown), which belongs to the yakuba complex, with high sequence homology to that of D. yakuba (Monnerot et al. 1990). Therefore, it appeared that duplication of these elements have occurred after the divergence of D. yakuba and a common ancestor of the melanogaster complex, and that the number of elements have subsequently increased or decreased. Without recombination, one of the mechanisms that might have generated such repetition of elements would be slippage during mtDNA replication (Buroker et al., 1990).

The NJ tree based on the alignment of the type II elements is shown in Fig. 6b. It is indicated in this tree that the innermost type II element of $D$. mauritiana (maII) accumulated many substitutions. In D. melanogaster, the outermost type II element accumulated more substitutions than the other elements. The elements adjacent to the T-stretch might be prone to accumulate substitutions by an as of yet unknown mechanism. The topology has been shown to differ from that of the coding sequences of mtDNA (Ballard, 2000). Since the sequences used in this analysis were only those elements around the T-stretch in other types of mtDNA, the reasons for this discrepancy in the topology would be difficult to be clarified.

Function of the T-stretch As described above, the innermost type II element was incomplete due to a large deletion, and there were short tandem repeats around the $\mathrm{T}$-stretch in the center of the A+T-rich region in most types of mtDNA in the melanogaster complex examined thus far. The complete sequences of the A+T-rich region in D. mauritiana (maII) and D. melanogaster further confirmed this observation. In D. melanogaster, the type I element and type II element in the center of the A+T-rich region contained more substitutions than did the other elements (Figs. 2 and 3). The innermost type II element was incomplete, and there were short repeats between the central T-stretch and the innermost type I element. It was also observed that in D. mauritiana (maII), the innermost type II element was incomplete, and that the innermost type I element contained short repeats.

Every type of mtDNA in the melanogaster complex has two T-stretches in different DNA strands within the A+Trich region, one of which is adjacent to the gene for tRNA $^{\text {ile, }}$, and the other in the center of the A+T-rich region. This has also been reported in studies of other flies (Lessinger and Azeredo-Espin, 2000) and even other insects (Zhang et al., 1995; Snall et al., 2002; Saitou et al., 2005). T-stretches are thought to be a conserved motif among insects and are most likely associated with some function of mtDNA. In D. obscura and other Drosophila, it was reported that mtDNA replication starts from $1 \mathrm{bp}$ downstream of the T-stretch (Saitou et al., 2005). Longley and co-workers (2001) suggested that errors caused by faulty polymerase $\gamma$, which functions in mtDNA replication, may be particularly prone to frame-shift mutations when homopolymeric runs in mtDNA are replicated. In the melanogaster complex, the short repeats around Tstretches might be generated by slippage in replication, and substitutions might occur due to polymerase $\gamma$ induced errors.

To gain a more precise understanding of the mechanism of concerted evolution in the A+T-rich region, and to clarify the function of the T-stretch, further comparative studies of the A+T-rich region in the melanogaster complex and in other species will be necessary.

\section{ACKNOWLEDGEMENTS}

This work was partially supported by a Grant-in-Aid for Scientific Research (C) (No. 15570004) from the Ministry of Education, Culture, Sports, Science and Technology, Japan to E. T. M.

\section{REFERENCES}

Ballard, J. W. O. (2000) Comparative genomics of mitochondrial DNA in members of the Drosophila melanogaster subgroup. J. Mol. Evol. 51, 48-63.

Brehm, A., Harris, D. J., Hernandez, M., Cabrera, V. M., Larruga, J. M., Pinto, F. M., and Gonzalez, A. M. (2001) Structure and evolution of the mitochondrial DNA complete control region in the Drosophila subobscura subgroup. Insect Mol. Biol. 10, 573-578.

Brown, T. A., and Clayton, D. A. (2002) Release of replication termination controls mitochondrial DNA copy number after depletion with 2',3'-dideoxycytidine. Nucleic Acids Res. 30, 2004-2010.

Buroker, N. E., Brown, J. R., Gilbert, T. A., O’Hara, P. J., Beckenbach, A. T., Thomas, W. K., and Smith, M. J. (1990). Length heteroplasmy of sturgeon mitochondrial DNA: an illegitimate elongation model. Genetics 124, 157-163.

Caccone, A., Garcia, B. A., and Powell, J. R. (1996) Evolution of the mitochondrial DNA control region in the Anopheles gambiae complex. Insect Mol. Biol. 1, 51-59.

Clary, D. O., and Wolstenholme, D. R. (1985) The mitochondrial DNA molecule of Drosophila yakuba: nucleotide sequence, gene organization, and genetic code. J. Mol. Evol. 22, 252- 
271.

Crozier, R. H., and Crozier, Y. C. (1993) The mitochondrial genome of the honeybee Apis mellifera : complete sequence and genome organization. Genetics 133, 97-117.

Fauron, C. M.-R., and Wolstenholme, D. R. (1976) Structural heterogeneity of mitochondrial DNA molecules within the genus Drosophila. Proc. Natl. Acad. Sci. USA 73, 36233627.

Goddard, J. M., and Wolstenholme, D. R. (1978) Origin and direction of replication in mitochondrial DNA molecules from Drosophila melanogaster. Proc. Natl. Acad. Sci. USA 75, 3886-3890.

Goddard, J. M., and Wolstenholme, D. R. (1980) Origin and direction of replication in mitochondrial DNA molecules from the genus Drosophila. Nucleic Acids Res. 8, 741-755.

Inohira, K., Hara, T., and Matsuura, E. T. (1997) Nucleotide sequence divergence in the A+T-rich region of mitochondrial DNA in Drosophila simulans and Drosophila mauritiana. Mol. Biol. Evol. 14, 814-822.

Kumar, S., Tamura, K., Jakobsen, I. B., and Nei, M. (2001) MEGA2: molecular evolutionary genetics analysis software. Bioinformatics 17, 1244-1245.

Lessinger, A. C., and Azeredo-Espin, A. M. (2000) Evolution and structural organization of mitochondrial DNA control region of myiasis-causing flies. Med. Vet. Entomol. 14, 71-80.

Lewis, D. L., Farr, C. L., Farquhar, A. L., and Kaguni, L. S. (1994) Sequence, organization, and evolution of the A+T region of Drosophila melanogaster mitochondrial DNA. Mol. Biol. Evol. 11, 523-538.

Longley, M. J., Nguyen, D., Kunkel, T. A., and Copeland, W. C. (2001) The fidelity of human DNA polymerase gamma with and without exonucleolytic proofreading and the p55 accessory subunit. J. Biol. Chem. 276, 38555-38562.

Monforte, A., Barrio, E., and Latorre, A. (1993) Characterization of the length polymorphism in the A+T-rich region of the Drosophila obscura group species. J. Mol. Evol. 36, 214223.

Monnerot, M., Solignac, M., and Wolstenholme, D. R. (1990) Discrepancy in divergence of the mitochondrial and nuclear genomes of Drosophila teissieri and Drosophila yakuba. J. Mol. Evol. 30, 500-508.

Nei, M., and Kumar, S. (2000) Evolutionary Change of DNA Sequences. In: Molecular Evolution and Phylogenetics, pp. 33-50. Oxford University Press, New York.
Rand, D. M., and Harrison, R. G. (1989) Molecular population genetics of mtDNA size variation in crickets. Genetics 121, 551-569.

Saccone, C., De Giorgi, C., Gissi, C., Pesole, G., and Reyes, A. (1999) Evolutionary genomics in Metazoa: the mitochondrial DNA as a model system. Gene 238, 195-209.

Saitou, N., and Nei, M. (1987) The neighbor-joining method: a new method for reconstructing phylogenetic trees. Mol. Biol. Evol. 4, 406-425.

Saitou, S., Tamura, K., and Aotsuka, T. (2005) Replication origin of mitochondrial DNA in insects. Genetics 171, 1695-1705.

Satta, Y., Ishiwa, H., and Chigusa S. I. (1987) Analysis of nucleotide substitutions of mitochondrial DNAs in Drosophila melanogaster and its sibling species. Mol. Biol. Evol. 4, 638-650.

Shadel, G. S., and Clayton, D. A. (1997) Mitochondrial DNA maintenance in invertebrates. Annu. Rev. Biochem. 66, $409-435$.

Snall, N., Huoponen, K., Savontaus, M. L., and Ruohomaki, K. (2002) Tandem repeats and length variation in the mitochondrial DNA control region of Epirrita autumnata (Lepidoptera: Geometridae). Genome 45, 855-861.

Solignac, M., Monnerot, M., and Mounolou, J.-C. (1986a) Mitochondrial DNA evolution in the melanogaster species subgroup of Drosophila. J. Mol. Evol. 23, 31-40.

Solignac, M., Monnerot, M., and Mounolou, J.-C. (1986b) Concerted evolution of sequence repeats in Drosophila mitochondrial DNA. J. Mol. Evol. 24, 53-60.

Thompson. J. D., Higgins, D. G., and Gibson, T. J. (1994) CLUSTAL W: improving the sensitivity of progressive multiple sequence alignment through sequence weighting, position-specific gap penalties and weight matrix choice. Nucleic Acids Res. 22, 4673-4680.

Tsujino, F., Kosemura, A., Inohira, K., Hara, T., Otsuka, F. Y., Obara, M. K., and Matsuura, E. T. (2002) Evolution of the $\mathrm{A}+\mathrm{T}$-rich region of mitochondrial DNA in the melanogaster species subgroup of Drosophila. J. Mol. Evol. 55, 573-583.

Zhang, D.-X., Szymura, J. M., and Hewitt, G. M. (1995) Evolution and structural conservation of the control region of insect mitochondrial DNA. J. Mol. Evol. 40, 382-391.

Zuker, M. (2003) Mfold web server for nucleic acid folding and hybridization prediction. Nucleic Acids Res. 31, 34063415. 